Supporting Information for:

\title{
Machine Learning for Vibrational Spectroscopic Maps
}

\author{
Alexei A. Kananenka, ${ }^{1,2}$ Kun Yao, ${ }^{3}$ Steven A. Corcelli, ${ }^{3}$ and J. L. Skinner ${ }^{1}$ \\ ${ }^{1}$ Pritzker School of Molecular Engineering, The University of Chicago, Chicago, Illinois 60637, USA \\ ${ }^{2}$ Department of Physics and Astronomy, University of Delaware, Newark, Delaware 19716, USA \\ ${ }^{3}$ Department of Chemistry and Biochemistry, University of Notre Dame, Notre Dame, Indiana 46556, USA
}


TABLE S1. Symmetry function parameters for hydrogen adopted from Morawietz et al. Proc. Natl. Acad. Sci. USA 2016, 113, 8368-8373. The cut-off radius $R_{c}=12$ Bohr.

\begin{tabular}{|c|c|c|c|c|c|c|c|}
\hline No. & Type & Element $j$ & Element $k$ & $R_{s}[\mathrm{Bohr}]$ & $\eta\left[\mathrm{Bohr}^{-2}\right]$ & $\lambda$ & $\zeta$ \\
\hline 1 & Radial & $\mathrm{H}$ & - & 0.0 & 0.001 & - & - \\
\hline 2 & Radial & $\mathrm{O}$ & - & 0.0 & 0.001 & - & - \\
\hline 3 & Radial & $\mathrm{H}$ & - & 0.0 & 0.010 & - & - \\
\hline 4 & Radial & $\mathrm{O}$ & - & 0.0 & 0.010 & - & - \\
\hline 5 & Radial & $\mathrm{H}$ & - & 0.0 & 0.030 & - & - \\
\hline 6 & Radial & $\mathrm{O}$ & - & 0.0 & 0.030 & - & - \\
\hline 7 & Radial & $\mathrm{H}$ & - & 0.0 & 0.060 & - & - \\
\hline 8 & Radial & $\mathrm{O}$ & - & 0.0 & 0.060 & - & - \\
\hline 9 & Radial & $\mathrm{O}$ & - & 0.9 & 0.150 & - & - \\
\hline 10 & Radial & $\mathrm{H}$ & - & 1.9 & 0.150 & - & - \\
\hline 11 & Radial & $\mathrm{O}$ & - & 0.9 & 0.300 & - & - \\
\hline 12 & Radial & $\mathrm{H}$ & - & 1.9 & 0.300 & - & - \\
\hline 13 & Radial & $\mathrm{O}$ & - & 0.9 & 0.600 & - & - \\
\hline 14 & Radial & $\mathrm{H}$ & - & 1.9 & 0.600 & - & - \\
\hline 15 & Radial & $\mathrm{O}$ & - & 0.9 & 1.500 & - & - \\
\hline 16 & Radial & $\mathrm{H}$ & - & 1.9 & 1.500 & - & - \\
\hline 17 & Angular & $\mathrm{O}$ & $\mathrm{O}$ & 0.0 & 0.001 & -1.0 & 4.0 \\
\hline 18 & Angular & $\mathrm{O}$ & $\mathrm{O}$ & 0.0 & 0.001 & 1.0 & 4.0 \\
\hline 19 & Angular & $\mathrm{H}$ & $\mathrm{O}$ & 0.0 & 0.010 & -1.0 & 4.0 \\
\hline 20 & Angular & $\mathrm{H}$ & $\mathrm{O}$ & 0.0 & 0.010 & 1.0 & 4.0 \\
\hline 21 & Angular & $\mathrm{H}$ & $\mathrm{O}$ & 0.0 & 0.030 & -1.0 & 1.0 \\
\hline 22 & Angular & $\mathrm{O}$ & $\mathrm{O}$ & 0.0 & 0.030 & -1.0 & 1.0 \\
\hline 23 & Angular & $\mathrm{H}$ & $\mathrm{O}$ & 0.0 & 0.030 & 1.0 & 1.0 \\
\hline 24 & Angular & $\mathrm{O}$ & $\mathrm{O}$ & 0.0 & 0.030 & 1.0 & 1.0 \\
\hline 25 & Angular & $\mathrm{H}$ & $\mathrm{O}$ & 0.0 & 0.070 & -1.0 & 1.0 \\
\hline 26 & Angular & $\mathrm{H}$ & $\mathrm{O}$ & 0.0 & 0.070 & 1.0 & 1.0 \\
\hline 27 & Angular & $\mathrm{H}$ & $\mathrm{O}$ & 0.0 & 0.200 & 1.0 & 1.0 \\
\hline
\end{tabular}


TABLE S2. Symmetry function parameters for oxygen adopted from Morawietz et al. Proc. Natl. Acad. Sci. USA 2016, 113, 8368-8373. The cut-off radius $R_{c}=12$ Bohr.

\begin{tabular}{|c|c|c|c|c|c|c|c|}
\hline No. & Type & Element $j$ & Element $k$ & $R_{s}$ & $\eta$ & $\lambda$ & $\zeta$ \\
\hline 1 & Radial & $\mathrm{H}$ & - & 0.0 & 0.001 & - & - \\
\hline 2 & Radial & $\mathrm{O}$ & - & 0.0 & 0.001 & - & - \\
\hline 3 & Radial & $\mathrm{H}$ & - & 0.0 & 0.010 & - & - \\
\hline 4 & Radial & $\mathrm{O}$ & - & 0.0 & 0.010 & - & - \\
\hline 5 & Radial & $\mathrm{H}$ & - & 0.0 & 0.030 & - & - \\
\hline 6 & Radial & $\mathrm{O}$ & - & 0.0 & 0.030 & - & - \\
\hline 7 & Radial & $\mathrm{H}$ & - & 0.0 & 0.060 & - & - \\
\hline 8 & Radial & $\mathrm{O}$ & - & 0.0 & 0.060 & - & - \\
\hline 9 & Radial & $\mathrm{H}$ & - & 0.9 & 0.150 & - & - \\
\hline 10 & Radial & $\mathrm{O}$ & - & 4.0 & 0.150 & - & - \\
\hline 11 & Radial & $\mathrm{H}$ & - & 0.9 & 0.300 & - & - \\
\hline 12 & Radial & $\mathrm{O}$ & - & 4.0 & 0.300 & - & - \\
\hline 13 & Radial & $\mathrm{H}$ & - & 0.9 & 0.600 & - & - \\
\hline 14 & Radial & $\mathrm{O}$ & - & 4.0 & 0.600 & - & - \\
\hline 15 & Radial & $\mathrm{H}$ & - & 0.9 & 1.500 & - & - \\
\hline 16 & Radial & $\mathrm{O}$ & - & 4.0 & 1.500 & - & - \\
\hline 17 & Angular & $\mathrm{H}$ & $\mathrm{O}$ & 0.0 & 0.001 & -1.0 & 4.0 \\
\hline 18 & Angular & $\mathrm{O}$ & $\mathrm{O}$ & 0.0 & 0.001 & -1.0 & 4.0 \\
\hline 19 & Angular & $\mathrm{H}$ & $\mathrm{O}$ & 0.0 & 0.001 & 1.0 & 4.0 \\
\hline 20 & Angular & $\mathrm{O}$ & $\mathrm{O}$ & 0.0 & 0.001 & 1.0 & 4.0 \\
\hline 21 & Angular & $\mathrm{H}$ & $\mathrm{H}$ & 0.0 & 0.010 & -1.0 & 4.0 \\
\hline 22 & Angular & $\mathrm{H}$ & $\mathrm{H}$ & 0.0 & 0.010 & 1.0 & 4.0 \\
\hline 23 & Angular & $\mathrm{H}$ & $\mathrm{H}$ & 0.0 & 0.030 & -1.0 & 1.0 \\
\hline 24 & Angular & $\mathrm{H}$ & $\mathrm{O}$ & 0.0 & 0.030 & -1.0 & 1.0 \\
\hline 25 & Angular & $\mathrm{O}$ & $\mathrm{O}$ & 0.0 & 0.030 & -1.0 & 1.0 \\
\hline 26 & Angular & $\mathrm{H}$ & $\mathrm{H}$ & 0.0 & 0.030 & 1.0 & 1.0 \\
\hline 27 & Angular & $\mathrm{H}$ & $\mathrm{O}$ & 0.0 & 0.030 & 1.0 & 1.0 \\
\hline 28 & Angular & $\mathrm{O}$ & $\mathrm{O}$ & 0.0 & 0.030 & 1.0 & 1.0 \\
\hline 29 & Angular & $\mathrm{H}$ & $\mathrm{H}$ & 0.0 & 0.070 & -1.0 & 1.0 \\
\hline 30 & Angular & $\mathrm{H}$ & $\mathrm{H}$ & 0.0 & 0.070 & 1.0 & 1.0 \\
\hline
\end{tabular}

TABLE S3. Total number of input and trainable neural network parameters.

Total number of symmetry functions

